\title{
Skin and Soft Tissue Infections in Plastic Surgery Over 10 Years
}

\author{
So-Eun Han, Sung Oh Hwang, Soo Hyang Lee, Pil Dong Cho \\ Department of Plastic and Reconstructive Surgery, Inje University Ilsan Paik Hospital, Inje University College of Medicine, Goyang, Korea
}

\begin{abstract}
Background: Skin and soft tissue infections (SSTIs) are frequently managed by plastic surgeons. Nowadays, antibiotics resistant organisms are increasing due to inappropriate use of antibiotics, and their treatment is becoming more complex. Regional data of microbiology, resistance patterns, and risk factors associated with resistance in specific types of infections are important in planning the management of SSTIs.

Methods: A 13-year (2004 to 2016) retrospective chart review of 229 patients with SSTI was performed to elucidate the trend of SSTI with respect to the pathogenic organisms, their antibiotic resistance, and treatment results.

Results: Staphylococcus aureus was a single, most dominant organism (40\%), and methicillin-resistant S. aureus (MRSA) was cultured in $80 \%$ of the cases. MRSA bacteria was detected significantly higher in patients who were admitted to a hospital within the past 12 months. Patients whose initial treatment failed received intravenous antibiotic therapy for a mean duration of 6.65 additional days; patients with successful initial treatment received no intravenous antibiotic therapy. Moreover, delayed use of vancomycin in patients with complicated SSTI showed a correlation with extended hospital stays and higher total costs.

Conclusion: The incidence of MRSA-related SSTI is increasing at a considerable rate, according to the retrospective examination of our data. When MRSA infection is suspected, empirical therapy should not be delayed, if culture report is obtained. This study may be helpful to plastic surgeons for treating SSTI.
\end{abstract}

Keywords: Skin and soft tissue infections; Methicillin-resistant Staphylococcus aureus; Antibiotics

\section{Introduction}

Skin and soft tissue infections (SSTIs) are usually observed in the field of plastic surgery, and often are reasons for patients to make a visit to hospitals [1]. There has been an alteration in the microbiology of SSTIs throughout the past decade. Prior to the 1990s, methicillin-sensitive Staphylococcus aureus (MSSA) was the common origin of SSTIs [2]. However, a number of recent surveillance studies noticed that communityacquired methicillin-resistant $S$. aureus (CA-MRSA) has appeared as the main SSTIs pathogen [3]. Since its discovery in the 1960s, MRSA has been identified as a hospital-acquired bacterium. More lately however, MRSA infections have been reported in unexposed patients [4]. Prior to the emergence of CA-MRSA, empirical use of $\beta$-lactam antibiotics was an appropriate treatment for most SSTIs, due to their effectiveness for treating Staphylococcus pyogenes and MSSA. In most countries, MRSA now occupies most SSTIs, and $\beta$-lactam antibiotics are not as effective as they once were [5]. MRSA-infected patients tend to have higher mortality rates, longer length of admission, and higher hospital cost than those with MSSA infections [6]. The most important contributing factor for resistance is abuse of broad-spectrum antibiotics. Moreover, there is a growing awareness that an early inappropriate antibiotic treatment leads to worsened outcomes [7]. Regional data of microbiology, resistance pat-

\section{Original Article}

Received: June 6, 2018

Revised: September 10, 2018

Accepted: October 13, 2018

\section{Corresponding author:}

Pil Dong Cho, M.D.

Department of Plastic and Reconstructive Surgery, Inje University Ilsan Paik Hospital, Inje University College of Medicine, 170 Juhwa-ro, Ilsanseo-gu, Goyang 10380, Korea

Tel: $+82-31-910-7322$

Fax: +82-31-910-7814

E-mail: pildong@naver.com

This is an Open Access article distributed under the terms of the Creative Commons Attribution Non-Commercial License (http://creativecommons.org/licenses/by-nc/4.0/) which permits unrestricted non-commercial use, distribution, and reproduction in any medium, provided the original work is properly cited.

(c) 2019 Korean Wound Management Society 
terns, and risk factors associated with resistance in specific types of infections are important for the assessment of the risk-benefit ratio among responsible use of proper empirical antibiotics and concerns for emergence of resistance

\section{Methods}

A retrospective review was conducted on all patients with SSTIs who were treated in a single institution over a 10 -year period (from January 2004 to December 2016). All patients with purulent SSTIs during this period underwent a wound swab for bacterial culture. At the department of laboratory medicine, the specimens were processed in accordance with the standard method. Antimicrobial susceptibility testing was executed by the disk diffusion method. Medical records were reviewed to assemble the demographic data of patients. Data collected included the type of infection, location, age, gender, comorbidities, pathogenic organisms, antibiotic sensitivity, treatment results, and total length of hospital stay (LOS). Patients with a history of previous hospitalization within the past 12 months, history of infection or colonization, admission to a nursing home, dialysis, surgery, or presence of permanent indwelling catheters (e.g., urinary catheter), or medical devices were classified in the nosocomial infection group [8]. Founded on these assembled data, patients with community-acquired infection were distinguished from nosocomial infected patients. SSTI classifications were comprised of superficial cellulitis, simple abscess, surgical device- or prosthesis-associated infection, surgical wound infection, diabetic foot ulcer, decubitus ulcer, and any other types of SSTI. Polymicrobial infection was considered present if more than one pathogen was determined as the reason of infection. Empirical treatment was categorized as inappropriate if there was a hold-up of more than 24 hours in initiation of therapy with antibiotics that show in vitro effects with identified pathogen(s).

Descriptive statistics, comparing patients who have MRSA infected SSTI with those who have non-MRSA infected SSTI, were performed. Student t-test for parametric distributions and Mann-Whitney U-test for non-parametric distributions were used to compare all continuous variables. We used a linear regression analysis to analyze the trends in annual consumption of antimicrobial agents and trends in incidence of pathogens over time. To adjust for potential confounding variables in multivariate analyses, we used logistic regression models. A P-value of $<0.05$ was considered statistically significant. All data were analyzed using PASW Statistics for Win- dows version 18.0 (SPSS Inc., Chicago, IL, USA).

\section{Results}

A total of 229 patients with a culture-positive specimen, which indicated SSTIs, were recruited. Patients were 0 to 93 years old, with a median age of 48 years. About three-fifths of patients (60\%) were male, and one-fifth of patient (20\%) were 30 to 39 years old. A total of $33.1 \%$ had superficial cellulitis or simple abscess, $14.4 \%$ had decubitus ulcer, $44.5 \%$ had surgical wound infection, $1.7 \%$ had surgical device-associated or prosthesis associated infection, $0.9 \%$ had diabetic foot ulcer, and 5.2\% had other SSTIs. Moreover, there was no significant difference between the MRSA infection group and non-MRSA infection group (Table 1). Infections were located on the lower extremity in 77 patients $(33.6 \%)$, head and neck in 52 patients (22.7\%), upper extremity in 35 (15.3\%), pressure sore site in $33(14.4 \%)$, and trunk in 32 (14\%). Seventy-seven patients (33.6\%) reported a history of trauma, 72 patients $(31.4 \%)$ reported a history of surgery, and 33 patients (14.4\%) reported a history of decubitus ulcer.

Infections were monomicrobial in 163 patients (71.2\%), and polymicrobial in 66 patients (28.8\%). S. aureus, coagulase negative Staphylococcus, and Pseudomonas aeruginosa were frequently isolated from patients with both monomicrobial and polymicrobial infections. Streptococcus infection was more prevalent among those with monomicrobial infection. Enterobacter, Enterococcus, and Escherichia coli were more frequently

Table 1. Patient baseline characteristics

\begin{tabular}{lccc}
\hline Infection & $\begin{array}{c}\text { MRSA } \\
\text { infection } \\
(\mathrm{n}=77)\end{array}$ & $\begin{array}{c}\text { Non-MRSA } \\
\text { infection } \\
(\mathrm{n}=152)\end{array}$ & P-value \\
\hline Age (yr) & $50.2 \pm 19.9$ & $46.8 \pm 21.8$ & 0.256 \\
Female sex & $27(35.1)$ & $65(42.8)$ & 0.385 \\
Type of infection & & & \\
$\quad$ Superficial cellulitis or simple & $18(23.4)$ & $58(38.2)$ & 0.067 \\
$\quad$ abscess & $14(18.2)$ & $19(12.5)$ & 0.285 \\
$\begin{array}{l}\text { Decubitus ulcer } \\
\text { Surgical wound infection }\end{array}$ & $40(51.9)$ & $62(40.8)$ & 0.232 \\
Device-associated infection & $2(2.6)$ & $2(1.3)$ & 0.488 \\
Diabetic foot ulcer & 0 & $2(1.3)$ & 0.314 \\
Others ${ }^{a)}$ & $3(3.9)$ & $9(5.9)$ & 0.527 \\
\hline
\end{tabular}

Values are presented as mean \pm SD or number (\%). MRSA, methicillin-resistant Staphylococcus aureus. ${ }^{a}$ O)ther infection types included bite wound, necrotizing fasciitis, etc. 
isolated from patients with polymicrobial infection. During the study period, a total of $96 \mathrm{~S}$. aureus isolates causing SSTIs were identified. Fig. 1 shows the trends in MRSA infected patients among the total SSTI patients. There was a significant increase in the incidence of MRSA infected patients over time (Fig. 1).

According to our chart review, a total of 172 patients (75.1\%) were determined to have a hospital-acquired infection. The remaining 57 patients (24.9\%) were determined to have community-acquired infection. Organism distribution differed between the hospital-acquired SSTI group and community-acquired SSTI group. Methicillin resistance was more common among S. aureus isolates from patients with hospital-acquired infection (88.7\%) than among isolates from patients with community-acquired infection (65.4\%). All gram-negative pathogens and Candida species were more often identified from patients with hospital-acquired SSTI, with the exception of Citrobacter species, which were more commonly observed among patients with community-acquired SSTI (Table 2).

The data results showed that the majority of $S$. aureus eventually acquired antibiotic resistance. Additionally, as in the case of Acinetobacter baumannii, the ratio of imipenem-resis$\operatorname{tant}$ A. baumannii was higher by $72.4 \%$. However, it was found that the ratios of vancomycin-resistant Enterococcus and imipenem-resistant $P$. aeruginosa were $12.5 \%$ and $14 \%$, respectively. This shows that Enterococcus and P. aeruginosa are to a degree less antibiotic resistant (Fig. 2).

Table 3 shows the risk factors of infections in both MRSA infected patients and non-MRSA infected patients. Among the 77 MRSA patients, the most common risk factors included recent hospitalization in 34 patients (44.2\%). Diabetes melli- tus-the second most common risk factor-was present in almost $20.8 \%$ of patients. Furthermore, cerebrovascular acci-

Table 2. Microbiology data

\begin{tabular}{|c|c|c|c|}
\hline Pathogen & $\begin{array}{l}\text { Hospital- } \\
\text { acquired } \\
\text { infection } \\
(n=172)\end{array}$ & $\begin{array}{l}\text { Community- } \\
\text { acquired } \\
\text { infection } \\
(n=57)\end{array}$ & P-value \\
\hline \multicolumn{4}{|l|}{ Gram positive } \\
\hline \multicolumn{4}{|l|}{ Staphylococcus species } \\
\hline Staphylococcus aureus & $37(21.5)$ & $19(33.3)$ & 0.118 \\
\hline MRSA & $30(17.4)$ & $10(17.5)$ & 0.987 \\
\hline Coagulase negative $S$. & $21(12.2)$ & $8(14.0)$ & 0.737 \\
\hline Streptococcus species & $8(5.3)$ & $7(12.3)$ & 0.051 \\
\hline Enterococcus faecalis & $5(2.9)$ & $2(3.5)$ & 0.822 \\
\hline \multicolumn{4}{|l|}{ Gram negative } \\
\hline Pseudomonas aeruginosa & $18(10.5)$ & $6(10.5)$ & 0.990 \\
\hline Escherichia coli & $3(1.7)$ & 0 & 0.319 \\
\hline Klebsiella species & $1(0.6)$ & $1(1.8)$ & 0.412 \\
\hline Proteus mirabilis & $2(1.2)$ & 0 & 0.416 \\
\hline Enterobacterspecies & $2(1.2)$ & 0 & 0.416 \\
\hline Acinetobacterspecies & $12(7.0)$ & 0 & 0.046 \\
\hline Citrobacterspecies & 0 & $1(1.8)$ & 0.082 \\
\hline \multicolumn{4}{|l|}{ Other } \\
\hline Candida species & $2(1.2)$ & 0 & 0.416 \\
\hline Other organisma) & $6(3.5)$ & $2(3.5)$ & 0.994 \\
\hline Polymicrobial & $55(32.0)$ & $11(19.3)$ & 0.122 \\
\hline
\end{tabular}

Values are presented as number (\%).

MRSA, methicillin-resistant Staphylococcus aureus.

${ }^{a}$ Other included Serratia marcescens $(n=2)$, Stenotrophomonas maltophilia $(\mathrm{n}=2)$, Pasteurella multocida $(\mathrm{n}=1)$, Providencia stuartii $(\mathrm{n}=1)$, and Proteus vulgaris $(\mathrm{n}=2)$.

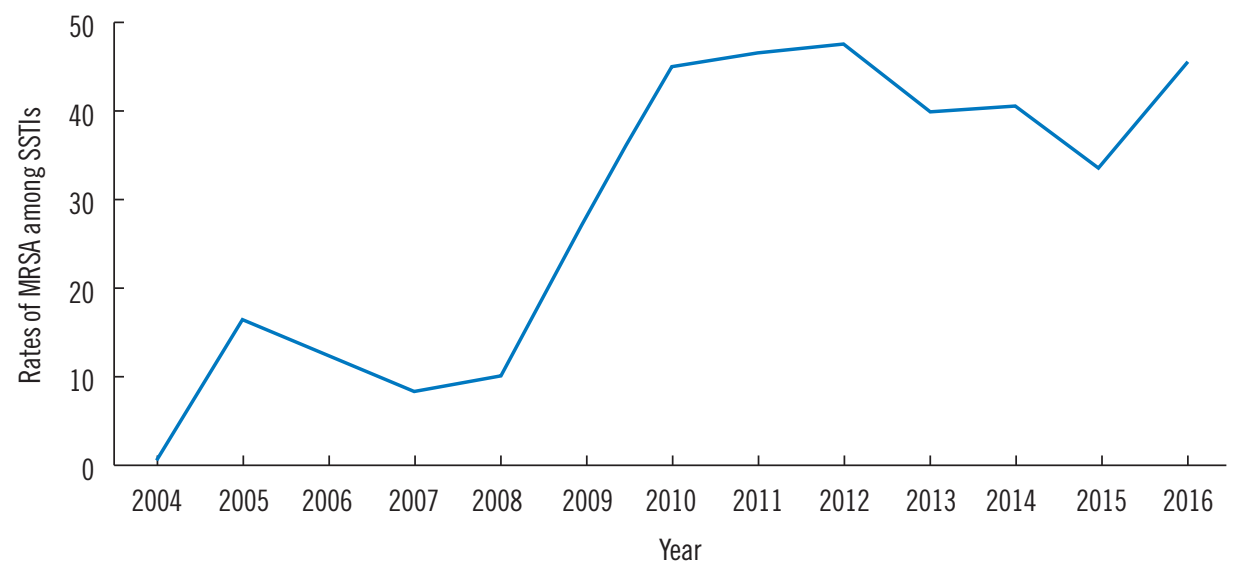

Fig. 1. Rates of methicillin-resistant Staphylococcus aureus (MRSA) among skin and soft tissue infections (SSTIs). Trends in MRSA infected patients among the total SSTI patients. There was a significant increase in the incidence of MRSA infected patients over time. 


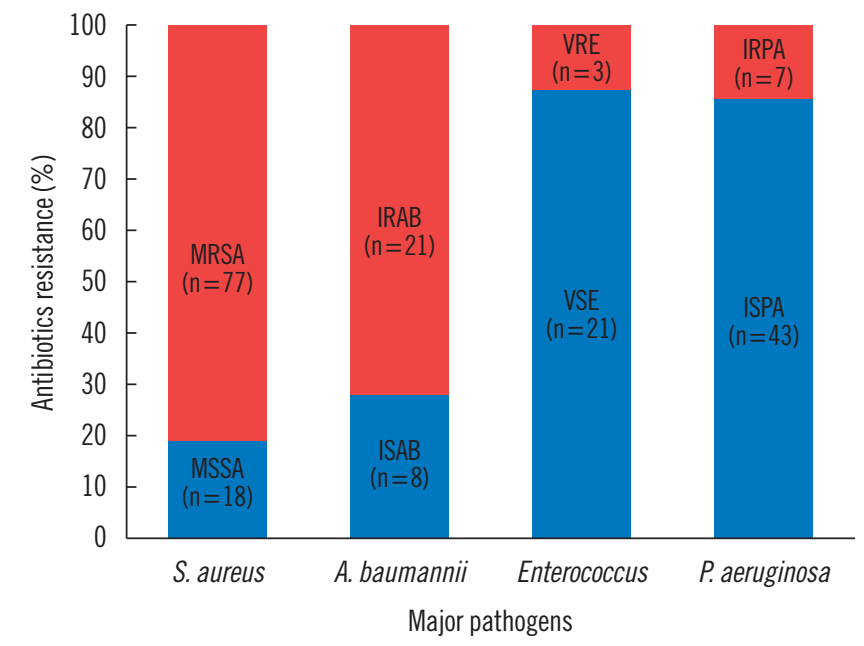

Fig. 2. Antibiotic resistance of microorganisms. MRSA, methicillin-resistant Staphylococcus aureus; MSSA, methicillin-sensitive S. aureus; IRAB, imipenem-resistant Acinetobacter baumannii; ISAB, imipenem-sensitive A. baumannii; VRE, vancomycin-resistant Enterococcus; VSE, vancomycin-sensitive Enterococcus; IRPA, imipenem-resistant Pseudomonas aeruginosa; ISPA, imipenem-sensitive $P$. aeruginosa.

dent was reported in 11 patients (14.3\%). Recent hospitalization is the only statistically significant risk factor for MRSA infection $(\mathrm{P}<0.001)$ (Table 3$)$.

Of the antibiotic therapy for 96 cultures containing $S$. aureus reviewed, 72 (75\%) were improper treatments, with a $\beta$-lactam antibiotic accounting for 56 (77.8\%) of these cases. Seventytwo (92.3\%) of 78 episodes with MRSA isolates were improperly treated with antibiotics. The antibiotic sensitivity pattern of MRSA in this group was $100 \%$ to vancomycin or teicoplanin, $95.2 \%$ to rifampin, $50 \%$ to sulfamethoxazole-trimethoprim (Bactrim), $14.1 \%$ to gentamicin, and $12.9 \%$ to tetracycline. The six appropriately treated cases of MRSA were treated with vancomycin (three cases), sulfamethoxazole-trimethoprim (Bactrim) (one case), teicoplanin (one case), and tetracycline (one case). Before microbiological cultures were obtained, the leading antibiotic category was $\beta$-lactams $(77.7 \%$ cephalosporins), which accounted for most of the prescriptions during the entire study period. After the culture report, the amount of antibiotics was reduced to $178(77.7 \%)$ to 71 (51.5\%) patients. In contrast to the case of cephalosporin, vancomycin usage was significantly increased from nine (3.9\%) to $53(23.1 \%)$.

The LOS differed substantially between the MRSA infection group and non-MRSA infection group. The mean LOS \pm standard deviation (SD) was $23.2 \pm 13.8$ days per patient. Among patients with MRSA infection, the mean LOS \pm SD was signifi-
Table 3. Risk factors associated with MRSA

\begin{tabular}{|c|c|c|c|}
\hline Risk factor & $\begin{array}{c}\text { MRSA } \\
\text { infection } \\
(n=77)\end{array}$ & $\begin{array}{c}\text { Non-MRSA } \\
\text { infection } \\
(n=152)\end{array}$ & P-value \\
\hline Recent hospitalization ${ }^{\text {a) }}$ & $34(44.2)$ & $29(19.1)$ & $<0.001$ \\
\hline Nursing home resident & $5(6.5)$ & $5(3.3)$ & 0.273 \\
\hline \multicolumn{4}{|l|}{ Comorbidities } \\
\hline Cerebrovascular accident & $11(14.3)$ & $19(12.5)$ & 0.724 \\
\hline Diabetes mellitus & $16(20.8)$ & $27(17.8)$ & 0.619 \\
\hline Peripheral vascular disease & $2(2.6)$ & $6(3.9)$ & 0.606 \\
\hline Liver disease & $5(6.5)$ & $5(3.3)$ & 0.273 \\
\hline Cancer & $3(3.9)$ & $4(2.6)$ & 0.605 \\
\hline Organ transplant & 0 & $1(0.7)$ & 0.477 \\
\hline Autoimmune disease & $2(2.6)$ & $2(1.3)$ & 0.488 \\
\hline End-stage renal disease & $2(2.6)$ & $4(2.6)$ & 0.988 \\
\hline
\end{tabular}

Values are presented as number (\%).

MRSA, methicillin-resistant $S$. aureus.

a) History of previous hospitalization within the past 12 months.

cantly longer $(26.3 \pm 12.0$ days vs. $21.7 \pm 14.5$ days; $\mathrm{P}<0.001)$ than those for patients with non-MRSA infection. Moreover, the mean $\mathrm{LOS} \pm \mathrm{SD}$ was significantly longer (27.3 \pm 11.9 days vs. $20.6 \pm 14.4$ days; $\mathrm{P}<0.001)$ in patients with inappropriate antibiotics use than those with appropriate antibiotic use.

\section{Discussion}

Until the 1970s, a variety of new antibiotics were introduced, which were effective for most common pathogens as the initial treatment. The final antibacterial drugs were developed during the 1980s. However, antimicrobial resistance has been increasing steadily, becoming a worldwide problem. The resistance progression is a natural evolutionary course for microorganisms; however, it is expedited by factors, such as high use of single agents, cross-transmission of resistance genes, suboptimal dosages, and poor isolation techniques [9].

In our retrospective cohort study, hospital-acquired infections occurred in $75.1 \%$ of patients. Hospital-acquired infection occurred in $27.2 \%$ of patients in a multi-institutional study by Lipsky et al. [10], and $73.6 \%$ of patients in a study by Zilberberg et al. [11]. This diversity is due to the differences in the population of study or criteria period that define a hospital-acquired infection. For example, the period prior to hospitalization or may be the difference between the previous antibiotic therapy is the reason. 
MRSA infections have appeared in the past few years as an important medical problem. We found that MRSA was present in a remarkable $33.6 \%$ of subjects, and $80.2 \%$ of all S. aureus isolates were MRSA. This is congruous with other studies reported throughout the past decade, which all suggested that the proportion of $S$. aureus isolates resistant to methicillin is on the rise [12]. Initially, MRSA was mainly a problem in hospital-acquired infections. Over the past 10 years, however, cases of community-acquired MRSA have been increasing considerably in some countries. Most reported proportions exceeded $20 \%$ in all World Health Organization regions, and even exceeded $80 \%$ in some reports [13]. The increasing incidence rate of methicillin resistance among community-acquired and hospital-acquired $S$. aureus isolates is faced with challenging decisions regarding the choice of antibiotic therapy, greater financial costs for persons with SSTI, and worse outcomes.

There are valid concerns about the economic influence of SSTI worldwide. Edelsberg et al. [14] examined the economic outcomes associated with the failure of initial treatment of SSTI. Patients who failed the initial treatment had a 3-fold higher risk of death in the hospital, and they incurred a mean hospital stay of additional 5.4 days. In the study by Zilberberg et al. [15], an inappropriate empiric therapy is associated with significantly prolonged hospital stay, by as much as 2 days. A total of 229 patients were evaluated for the appropriateness of the first antibiotic treatment on the basis of culture outcome acquired, including 140 who received appropriate therapy (61.1\%) and 89 who did not (38.9\%). Patients with unsuccessful first antibiotic therapy had an average of additional 6.65 days of intravenous antibiotic treatment compared with those with successful first antibiotic therapy. Infection with antibiotic-resistant isolates can lead to unsatisfactory outcomes, prolonged LOS, and increased cost of treatment [16]. In a comparative study concerning MRSA and MSSA, patients with MRSA infections had worse clinical and economic results $[15,17,18]$. Besides, delayed use of vancomycin in patients with complicated SSTI appears to be associated with extended hospital stay and more expensive total costs [19]. Therefore, a rapid diagnosis and proper treatment of MRSA are highly important for improving outcomes.

This study was designed to objectively quantify the incidence of antibiotic resistance microorganisms associated with SSTIs treated in a single institution. It was also aimed to help identify risk factors among patients to allow for immediate start of empiric antibiotic treatment of MRSA, without having to wait for culture results. If antibiotics were started without sending the materials for culture, then an evaluation of risk factors and local prevalence of MRSA should be considered. In this study, when analyzing the demographic characteristics (age, sex, infection type, risk factors, etc.) of patients with MRSA and non-MRSA, MRSA bacteria detected a statistically significant higher for patients who were recently admitted to hospitals within the last 12 months.

Our ability to find other risk factors for MRSA was limited by the retrospective nature of this study and incomplete medical records. In this study, local prevalence of MRSA was 33.6\%, and some authors recommend treatment coverage for MRSA when the local prevalence rate is greater than 15\% [20]. Therefore, we have to modify our institutional guidelines for treating SSTIs. For hospitalized patients with a complicated or severe SSTI, vancomycin or newer antistaphylococcal antibiotics (quinupristin-dalfopristin, linezolid, tigecycline, and daptomycin) should be considered while awaiting culture results. In our hospital, vancomycin, teicoplanin and rifampin can be used. A $\beta$-lactam antibiotic can be used in hospitalized patients with nonpurulent cellulitis, and the regimen can be modified specifically to MRSA-active therapy if there is no clinical reaction.

There are some limitations to consider. First, given the retrospective nature of this study, there may be biases, especially, selection bias. To reduce such possibilities, we have established an a priori case definitions and have registered successive patients for a specific period of time. The wound culture was obtained through a swab, which in effect indicated colonization rather than infection. Thus, resulting in an exaggerated rate of improper treatment, requiring longer follow-up period. Third, this study lacks data about the mortality of patients and post hospitalization outcomes, limiting itself to only the LOS.

MRSA-related SSTIs are increasing at a significant rate. These patients are more likely to be exposed to improper initial antibiotic therapy. Antibiotic therapy guidelines must consider this change to minimize the cost and morbidity, while maximizing success. We recommend that practitioners execute a culture-based treatment for all SSTIs and analyze the MRSA trends in their own society to adapt local therapy regimens for empiric MRSA coverage.

\section{Conflict of interest}

No potential conflicts of interest relevant to this article are reported. 


\section{References}

1. Hersh AL, Chambers HF, Maselli JH, et al. National trends in ambulatory visits and antibiotic prescribing for skin and soft-tissue infections. Arch Intern Med 2008;168:1585-91.

2. Abrahamian FM, Talan DA, Moran GJ. Management of skin and soft-tissue infections in the emergency department. Infect Dis Clin North Am 2008;22:89-116.

3. Chen LF, Chastain C, Anderson DJ. Community-acquired methicillin-resistant Staphylococcus aureus skin and soft tissue infections: management and prevention. Curr Infect Dis Rep 2011;13:442-50.

4. Moran GJ, Krishnadasan A, Gorwitz RJ, et al. Methicillin-resistant S. aureus infections among patients in the emergency department. N Engl J Med 2006;355:666-74.

5. Stevens DL, Bisno AL, Chambers HF, et al. Practice guidelines for the diagnosis and management of skin and soft tissue infections: 2014 update by the Infectious Diseases Society of America. Clin Infect Dis 2014;59:e10-52.

6. Skiest DJ, Brown K, Cooper TW, et al. Prospective comparison of methicillin-susceptible and methicillin-resistant community-associated Staphylococcus aureus infections in hospitalized patients. J Infect 2007;54:427-34.

7. Schramm GE, Johnson JA, Doherty JA, et al. Methicillin-resistant Staphylococcus aureus sterile-site infection: the importance of appropriate initial antimicrobial treatment. Crit Care Med 2006;34:2069-74.

8. Kiran RV, McCampbell B, Angeles AP, et al. Increased prevalence of community-acquired methicillin-resistant Staphylococcus aureus in hand infections at an urban medical center. Plast Reconstr Surg 2006;118:161-6.

9. LeBlanc DM, Reece EM, Horton JB, et al. Increasing incidence of methicillin-resistant Staphylococcus aureus in hand infections: a 3-year county hospital experience. Plast Reconstr Surg 2007;119:935-40.

10. Lipsky BA, Weigelt JA, Gupta V, et al. Skin, soft tissue, bone, and joint infections in hospitalized patients: epidemiology and microbiological, clinical, and economic outcomes. Infect Control Hosp Epidemiol 2007;28:1290-8.

11. Zilberberg MD, Shorr AF, Micek ST, et al. Epidemiology and outcomes of hospitalizations with complicated skin and skinstructure infections: implications of healthcare-associated infection risk factors. Infect Control Hosp Epidemiol 2009; 30:1203-10.

12. Rennie RP, Jones RN, Mutnick AH, et al. Occurrence and antimicrobial susceptibility patterns of pathogens isolated from skin and soft tissue infections: report from the SENTRY Antimicrobial Surveillance Program (United States and Canada, 2000). Diagn Microbiol Infect Dis 2003;45:287-93.

13. World Health Organization. Antimicrobial resistance: global report on surveillance [Internet]. Geneva: WHO; c2014 [cited 2019 Feb 10]. Available from: www.who.int/iris/bitstre am/10665/112642/1/9789241564748_eng.pdf.

14. Edelsberg J, Berger A, Weber DJ, et al. Clinical and economic consequences of failure of initial antibiotic therapy for hospitalized patients with complicated skin and skin-structure infections. Infect Control Hosp Epidemiol 2008;29:1609.

15. Zilberberg MD, Shorr AF, Micek ST, et al. Hospitalizations with healthcare-associated complicated skin and skin structure infections: impact of inappropriate empiric therapy on outcomes. J Hosp Med 2010;5:535-40.

16. Saravolatz LD, Markowitz N, Arking L, et al. Methicillin-resistant Staphylococcus aureus. Epidemiologic observations during a community-acquired outbreak. Ann Intern Med 1982; 96:11-6.

17. Kopp BJ, Nix DE, Armstrong EP. Clinical and economic analysis of methicillin-susceptible and -resistant Staphylococcus aureus infections. Ann Pharmacother 2004;38:1377-82.

18. Engemann JJ, Carmeli Y, Cosgrove SE, et al. Adverse clinical and economic outcomes attributable to methicillin resistance among patients with Staphylococcus aureus surgical site infection. Clin Infect Dis 2003;36:592-8.

19. Itani KM, Akhras KS, Stellhorn R, et al. Outcomes associated with initial versus later vancomycin use in patients with complicated skin and skin-structure infections. Pharmacoeconomics 2009;27:421-30.

20. Kaplan SL, Hulten KG, Gonzalez BE, et al. Three-year surveillance of community-acquired Staphylococcus aureus infections in children. Clin Infect Dis 2005;40:1785-91. 